\title{
DETERMINAÇÃO DA TOPOGRAFIA DE UMA FRUTA PELA TÉCNICA DE MOIRÉ DE SOMBRA COM MULTIPLICAÇÃO DE FRANJAS ${ }^{1}$
}

\author{
Fruit profilometry based on shadow Moiré techniques \\ Antonio Carlos Loureiro Lino², Inácio Maria Dal Fabbro ${ }^{3}$
}

\begin{abstract}
RESUMO
As técnicas de moiré (TM) são um conjunto de técnicas, baseadas no fenômeno de moiré, mais comumente utilizadas em perfilometria, devido à sua simplicidade e rapidez de medição. O fenômeno de moiré consiste na interferência causada pela sobreposição de duas estruturas periódicas (retículos), sendo a TM de sombra com multiplicação de franjas mais sensível que os métodos tradicionais, além de diminuir os efeitos da diferença de iluminação na superfície do objeto em estudo. Objetivou-se com este trabalho gerar um Modelo Digital de Elevação (MDE) de uma pêra pela TM de sombra com multiplicação de franjas e compará-lo a um outro modelo gerado a partir de um scanner 3D. Para este trabalho, foram utilizados um modelo de uma pêra, em escala 1:1, confeccionado em parafina e um esquema experimental constituído de um retículo de moiré, iluminador e câmera fotográfica digital. Pelos resultados, verifica-se uma alta correlação entre os MDE obtidos pelos dois métodos. A TM de sombra com multiplicação de franjas gerou um MDE que reproduz satisfatoriamente a topografia da pêra.
\end{abstract}

Termos para indexação: Técnicas de moiré, moiré de sombra, perfilometria, frutos.

\begin{abstract}
The Moiré Techniques (MT) are a group of techniques based on the moiré phenomenon, which are commonly used in profilometry, due to your simplicity and measurement speed. The moiré phenomenon consists of the interference caused by the overlapping of two periodic structures or grids. Shadow TM with multiplication of fringes is more sensitive than the traditional method and besides, they reduce the effects of the illumination difference in the surface of the object in study. The objective of this work was to generate a digital model of the surface of the pear for shadow TM with multiplication of fringes and to compare with another model generated starting from scanner 3D. For this, a model of a pear, in scale 1:1, made in paraffin and an experimental set up outline with moiré grid, light and digital photographic camera were used. The results showed a high correlation among the results from the two methods. Shadow TM with multiplication of fringes gave a satisfactory determination of topography of the pear.
\end{abstract}

Index terms: Moiré techniques, shadow moiré, perfilometry, fruits.

(Recebido para publicação em 10 de maio de 2002 e aprovado em 21 de outubro de 2002)

\section{INTRODUÇÃO}

A utilização de técnicas perfilométricas é de grande importância para diversos segmentos da indústria, biologia, medicina e engenharia, principalmente no que se refere a práticas de controle de qualidade, modelagem matemática e diagnósticos clínicos.

Em Engenharia Agrícola, depara-se com vários tipos de problemas que envolvem o estudo do perfil topografico de superfícies irregulares, tais como: órgãos vegetais (frutos e tubérculos), superfície do solo, elementos de máquinas. Com base no perfil topográfico, pode-se gerar um Modelo Digital de Elevação (MDE) desta superfície.

O MDE de órgãos vegetais pode ser utilizado no estudo do comportamento mecânico quando submetido à vibração, impactos, empilhamento e resfriamento, e ainda na seleção de frutos e no desenvolvimento de embalagens. Já o MDE da superfície do solo pode ser aplicado no controle de qualidade de operações de preparo de solo, com base na determinação do microrrelevo ou rugosidade superficial e ainda em estudos de erosão. No caso de elementos de máquinas, o MDE pode ser utilizado em engenharia reversa, no controle de qualidade de fabricação, no estudo de desgaste e deformação de peças ativas durante as operações agrícolas.

$\mathrm{Hu}$ (2001) afirma que as Técnicas de Moiré (TM) são as técnicas perfilométricas mais comumente utilizadas, em razão, principalmente, da sua simplicidade e rapidez de medição, e, por isso, elas têm sido alvo de freqüentes estudos e vários tipos de aplicações.

1. Trabalho é parte da dissertação de Mestrado em Engenharia Agrícola.

2. Engenheiro Agrícola, MsC, Pesquisador Científico, Centro Avançado de Engenharia e Automação Agrícola do IAC, Caixa Postal 26 - $13201-970$ Jundiaí, SP. acllino@ig.com.br

3. Engenheiro Agrônomo, PhD, Professor FEAGRI/UNICAMP, Departamento de Máquinas Agrícolas, Caixa Postal 6011 - $13081-490$ - Campinas, SP. inácio@agr.unicamp.br 
Oster e Nishijima (1964) afirmam que as TM baseiam-se no fenômeno de moiré. Esse fenômeno consiste na interferência causada pela sobreposição de duas estruturas periódicas ou retículos, tais como linhas paralelas ou radiais, círculos ou elipses concêntricas ou mesmo pontos que geram padrões característicos conhecidos como franjas de moiré.

Takasaki (1970) utilizou a interferência entre um retículo e a sua própria sombra projetada na superfície (moiré de sombra) para medir o relevo de objetos e pessoas. Nesse caso, as franjas de moiré formadas são constituídas por um conjunto de pontos de mesma cota, semelhantes às curvas de nível de mapas topográficos.

Moore e Truax (1979) afirmam que a exatidão para a TM de sombra é no máximo $1 / 10$ do período da franja de moiré. Já Dirckx e Decraemer (1990) afirmam que a exatidão da técnica depende da calibração do arranjo experimental, e que é possível saber perfeitamente a distância entre duas franjas de moiré.

Post et al. (1994) dizem que existem várias técnicas que permitem diminuir o erro das medições, entre elas a multiplicação de franjas, que é muito robusto, pois produz um maior número de franjas de moiré e contorna as dificuldades causadas pela variação da intensidade da iluminação ao longo do objeto em estudo, principalmente na TM de sombra. Esse método consiste em obter duas imagens das franjas de moiré defasadas uma da outra de I, ou seja, meio período da franja de moiré, de maneira que, se em uma imagem, no local onde existe uma franja clara, na outra imagem existirá uma franja escura, isto é, uma imagem é inversa da outra.

Willmot et al. (1985) declaram que a precisão é dada pelo coeficiente de correlação de Pearson o $R^{2}$ indica apenas o grau de dispersão dos dados obtidos, ou seja, quanto eles se ajustam a uma linha de tendência central. Ele considera apenas o erro aleatório. Já a exatidão, erro sistemático, das medidas é quantificada numericamente pelo índice de concordância (d) que indica num gráfico de dispersão, ou 1/1, o quanto esses dados se ajustam numa linha que, partindo da origem, divide o gráfico em duas partes iguais.
Realizou-se este trabalho com o objetivo de gerar um Modelo Digital de Elevação (MDE) de uma pêra pela TM de sombra com multiplicação de franjas e compará-lo a um outro modelo gerado a partir de um scanner 3D.

\section{MATERIAL E MÉTODOS}

Para a execução do trabalho, foi tomada a metade de réplica de uma pêra confeccionada em parafina. Em escala 1:1, a réplica trazia as dimensões 79.5 x 65,9 x 32,4 mm. A utilização de um espécime "in natura" foi descartada devido à sua alta perecibilidade. O objeto de estudo foi pintado com tinta branca fosca, a fim de proporcionar maior contraste das franjas de moiré e de facilitar a digitalização pelo Scanner 3D Laser.

Para a obtenção das imagens com as franjas de moiré, montou-se o esquema experimental apresentado na Figura 1, com os seguintes componentes:

- Fonte de luz: projetor de "slides" com lâmpada de $500 \mathrm{~W}$ de potência.

- Câmera fotográfica digital Kodak DC 120.

- Retículo de moiré com período de 0,5 mm, confeccionado em fotolito para proporcionar maior nitidez das linhas. Esse foi colocado entre duas placas de vidro de 100x100x3 mm, para evitar a sua deformação, sendo sustentado por um suporte metálico.

- Mecanismo que permite o deslocamento da pêra, afastando ou aproximando-a do retículo de Moiré.

Sendo:

"d" a distância entre a câmera e a fonte de luz,

"h" a distância entre a câmera e o retículo e

"p" o período do retículo.

Foram obtidas quatro imagens das franjas de moiré, com 256 tons de cinza, que variam de 0 (preto) a 255 (branco). Entre a tomada de cada uma das imagens, o objeto foi afastado do retículo de moiré, distâncias que produzam deslocamentos das fases das franjas de Moiré de $0, \mathbb{\Phi} / 4, \mathbb{q} / 2$ e 3ף/4. Como resultado, as imagens I1 e I3 são inversas entre si, isto é, se em um local da imagem I1 existe uma é franja clara, no mesmo local da imagem I3, a franja é escura. O mesmo ocorrendo com as imagens I2 e I4. 


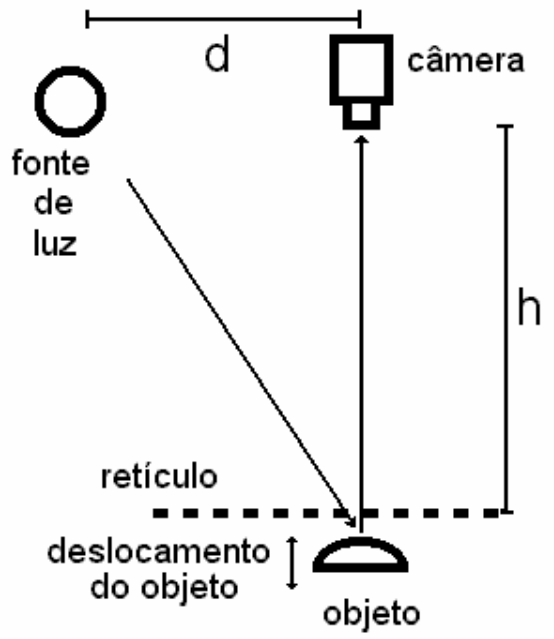

FIGURA 1 - Arranjo experimental para verificação da consistência do TM de sombra.

Os pares de imagens (I1 e I3 e I2 e I4) passaram pelo seguinte processo:

1. Filtragem em programa de edição de fotos (Corel PHOTO-PAINT 9) para a equalização (por meio do comando imagem/ajustar/equalização local) e remoção do retículo de moiré (por meio do comando efeitos/desfocar/passa-baixa).

2. Cálculo do módulo da subtração (programa IDRISI 32), para duplicar e evidenciar as franjas da superfície da pêra.

3. Reclassificação ou truncamento, atribuído-se 0 (preto) aos valores entre 0 e 30 (escuros) e 1 (branco) os valores acima de 30 (mais claros), gerando imagens $\left(\mathrm{I}_{1-3}\right.$ e $\left.\mathrm{I}_{2-4}\right)$ em branco e preto, com linhas delgadas, que correspondem às franjas de Moiré, facilitando a sua digitalização e eliminando os ruídos.

As imagens $\mathrm{I}_{1-3}$ e $\mathrm{I}_{2-4}$ foram multiplicadas produzindo uma imagem com um número quatro vezes maior de linhas que uma imagem de Moiré clássica.

A geração do Modelo Digital de Elevação (MDE) da pêra baseou-se na metodologia descrita por Valeriano (1999), que a utilizou para a geração de Modelos Digitais de Elevação (MDE) de terrenos a partir da digitalização das curvas de nível de mapas topográfi$\cos$.

A imagem com as franjas de moiré foi transferida para o programa AUTOCAD $2000^{\circledR}$, no qual foram então digitalizadas manualmente, criando vetores a espaçamentos de 1 ordem de franja (centro das franjas escuras) e para cada vetor, foram atribuídos valores de co- ta calculados pela equação proposta por Meadows et al. (1970).

$$
\Delta z=\frac{N p h}{d} \quad \text { Equação 1 }
$$

em que:

$\Delta z=$ a diferença de cota entre dois pontos na superfície do objeto

$N=$ número de franjas entre esses dois pontos

$d=$ distância da câmera à fonte de luz $(300 \mathrm{~mm})$

$h=$ distância da câmera ao retículo $(150 \mathrm{~mm})$

$p=$ período do retículo $(1 \mathrm{~mm})$

Obs: Os valores obtidos para $\Delta z$ foram divididos por 4 , pois a multiplicação de franjas produz 4 vezes mais franjas que o moiré clássico.

Esses vetores no formato ".DXF" contêm as coordenadas $\mathrm{x}$, y e $\mathrm{z}$ dos pontos que compõem as franjas digitalizadas. Utilizando-se o programa de conversão "DXF2DAT", foi criado um arquivo no formato ASCII e com extensão ".DAT”. O uso de uma planilha de cálculo permitiu o ajuste das escalas, eixos e pontos de origem das franjas de moiré.

O arquivo ".DAT" corrigido é tratado pelo programa SURFER $32^{\circledR}$, no qual foi interpolado, gerando um Modelo Digital de Elevação (MDE) da pêra.

Para a geração do MDE padrão, foi utilizado um perfilometro 3D composto de um scanner laser marca 3D SCANNERS, modelo ModelMaker. acoplado a um braço de posicionamento marca FARO, modelo Faro- 
Arms Silver, destinado ao posicionamento do scanner. Esse sistema produz medidas com um erro de 0,003 $\mathrm{mm}$.

Para possibilitar a comparação entre os dois modelos, o MDE da pêra gerado pelo Scanner 3D foi transferido para programa SURFER $32^{\circledR}$, para seu redimensionamento com os mesmos números de linhas e colunas daquele produzido pela TM.

Para a confrontar os métodos, foram extraídos 9 perfis longitudinais dos dois MDE, e o primeiro perfil passa pelo centro da pêra, e os demais, paralelos a este, 4 à esquerda e 4 à direita, distanciados $5 \mathrm{~mm}$ entre si (Figura 2).

Esses perfis foram tratados em planilha eletrônica e comparados por meio de análises Correlação, Regressão e pelo índice de concordância " $d$ " proposto por Willmot (1985).

\section{RESULTADOS E DISCUSSÃO}

$\mathrm{Na}$ Figura 3 verificam-se as imagens $\left(A=I_{1}\right.$, $\mathrm{B}=\mathrm{I}_{2}, \mathrm{C}=\mathrm{I}_{3}$ e $\left.\mathrm{D}=\mathrm{I}_{4}\right)$ da pêra, filtradas para a remoção das linhas de moiré, com 256 tons de cinza, em que 0 corresponde ao preto e 255, ao branco. Entre a aquisição de duas imagens, a pêra foi afastada do retículo de moiré, de modo que as franjas se deslocaram $1 / 4$ de período. As imagens $\mathrm{I}_{1}, \mathrm{I}_{2}, \mathrm{I}_{3}$ e $\mathrm{I}_{4}$ correspondem ao deslocamento das franjas de moiré de $0, \pi / 2, \pi$ e $3 \pi / 4$. Nota-se nessas imagens que as franjas de moiré aparecem apenas no topo da pêra. Nas regiões onde a declividade da superfície é alta, não há formação das franjas, como observado por Gasvik (1983), significando que a $\boldsymbol{T M}$ de sombra deve ser utilizada apenas onde a superfície do objeto em estudo é relativamente plana, ou para pequenas áreas de cada vez.

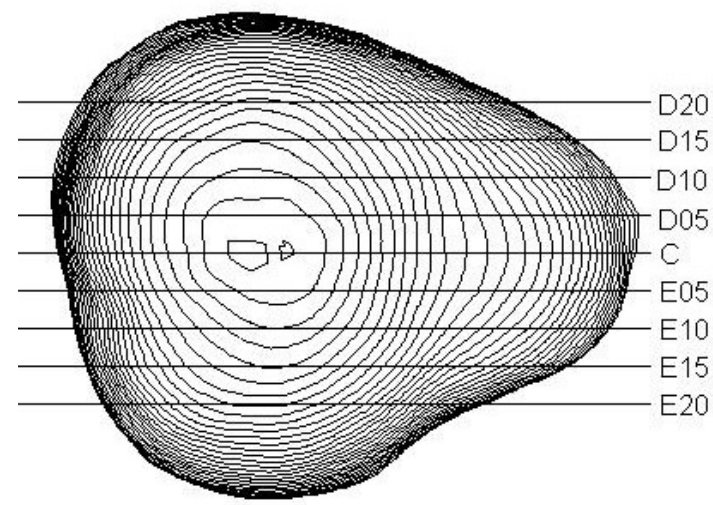

FIGURA 2 - Posicionamento dos perfis usados para comparar os MDE produzidos pela TM e pelo Scanner 3D Laser.
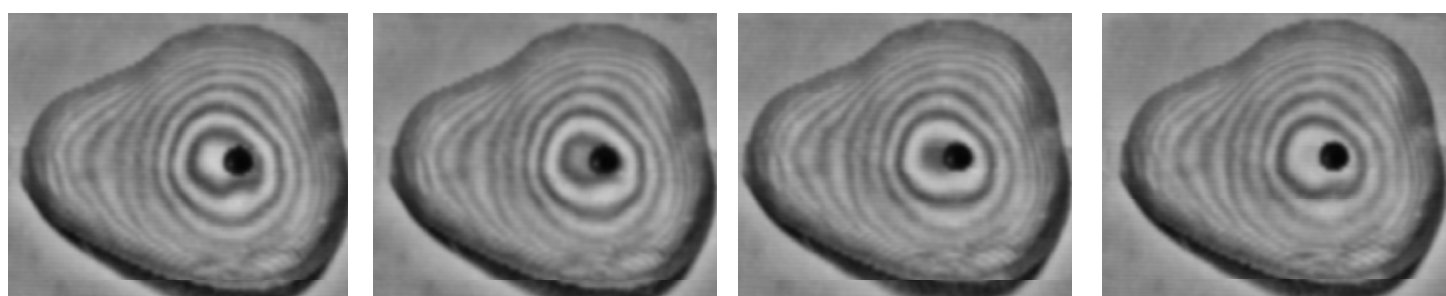

FIGURA 3 - Imagens filtradas da pêra. A. Imagem inicial (I1). B. Imagem com franjas defasadas $\pi / 2$ (I2). C. Imagem com franjas defasadas $\pi$ (I3). D.Imagem com franjas defasadas $3 \tilde{\pi} / 4$ (I4). 
A área circular preta no centro da figura corresponde a um furo feito na mesma para indicar o centro de massa da pêra, e corresponde às coordenadas $(0,0)$, ou seja, à origem dos eixos $\mathrm{x}, \mathrm{y}$.

Na Figura 4A observa-se a imagem resultante do módulo da subtração das imagens I1 e I3 e na Figura 4B verifica-se o truncamento dessa imagem, em que as linhas pretas correspondem, em tons de cinza, a valores de 0 a 15, e a área branca corresponde a valores de 15 a 255. Com isso, duplicou-se o número de franjas de moiré, eliminaram-se ruídos e equalizou-se a imagem, isto é, eliminou a influencia do reflexo da iluminação sobre a pêra, que prejudica a digitalização automática das franjas de moiré. O mesmo processo foi repetido para as imagens I2 e I4 (Figuras 4C e 4D).

As Figuras 4B e 4D foram mescladas, gerando a Figura 4E , que possui o quádruplo de franjas de moiré comparadas às imagens originais. Na Figura $4 \mathrm{~F}$ verificam-se as franjas de moiré digitalizadas no Programa AUTOCAD $2000^{\circledR}$

Nas Figuras 5 e 6 verificam-se os MDE gerados pelo Programa SURFER $32^{\circledR}$, mediante informações produzidas pelo Scanner 3D e TM. Comparando as Figuras 5A e 6A, nota-se que a TM gerou o MDE apenas da região superior ou topo da pêra, onde se podiam distinguir e digitalizar as franjas de moiré.

Observa-se nas Figuras 5A e 5B que nos cantos da imagem, onde não havia franjas de moiré, o programa gerou superfícies que na realidade não existem e que poderiam ocasionar erros. Isso é uma característica do interpolador do programa SURFER $32^{\circledR}$. Para contornar esse tipo de problema, poderia se utilizar outro programa para fazer a interpolação ou estudar a topografia do objeto, dividindo-o em pequenas regiões onde a topografia é relativamente plana.

Na Figura 7 encontra-se a sobreposição do perfil central produzido pela TM e pelo Scanner 3D. Considerou-se apenas a parte central da pêra, que é a mais plana, onde as franjas de moiré eram nítidas e puderam ser digitalizadas. Nessa região considerada, houve uma boa ajustagem entre os dados.

Na Tabela 1 apresentm-se altos valores de Correlação, $\mathrm{R}^{2}$ e índice de concordância de Willmott $(d)$ para o perfil central e os dois extremos. Os valores de $\mathrm{R}^{2}$ foram superiores a 0,93 , inferindo-se que os dados tiveram uma alta ajustagem à linha de tendência central, isto é, tiveram baixa dispersão ou alta precisão, o que significa que os erros aleatórios foram baixíssimos. O índice $d$ também apresentou valores elevados acima de 0,94 , significando que os erros sistemáticos também foram baixíssimos, apresentando uma alta exatidão.

Para os perfis externos, D20 e E20, o índice de concordância de Willmott $(d)$ apresentou uma tendência de queda. Isso se deve provavelmente à baixa visualização das franjas de moiré causada pelo aumento da declividade da superfície, como comentado por Gasvik (1983). Com o que concorda Valeriano (1999), que afirma que, para mapas topográficos, o erro da altura é diretamente proporcional à tangente da declividade. Quanto maior a declividade, maior será o erro médio da cota $\mathrm{z}$.
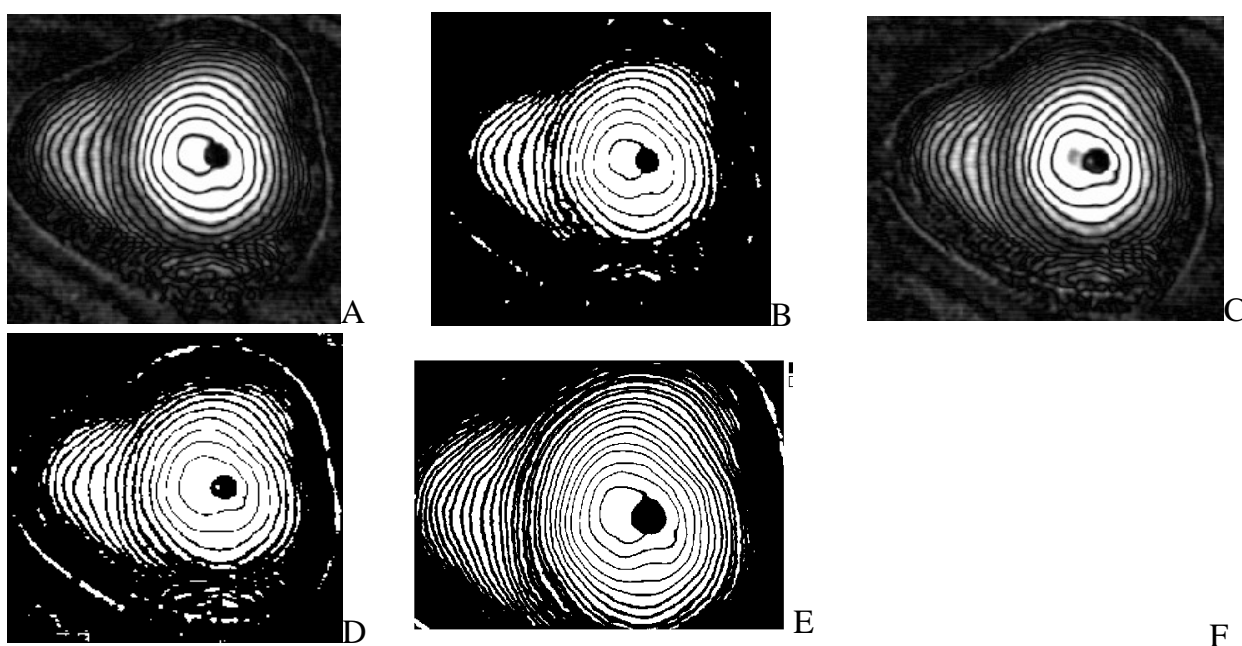

FIGURA 4 - Processo de multiplicação de franjas de moiré. A- Subtração de I1 e I3. B- Truncamento (I1-I3). C- Subtração de I2 e I4. D- Truncamento (I2-I4). E- Imagens truncadas (B e D) mescladas. F- Franjas de moiré digitalizadas. 
A

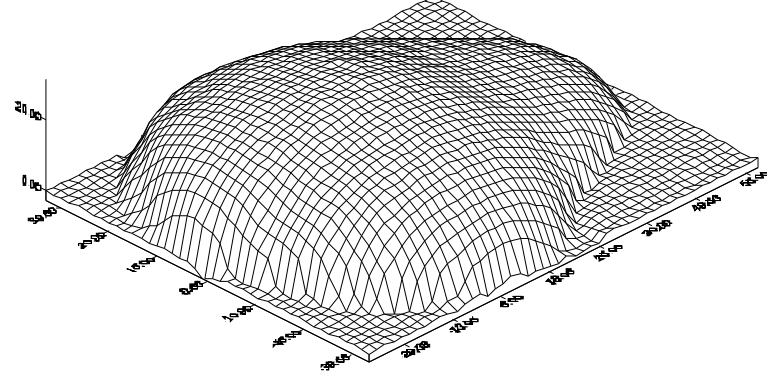

B

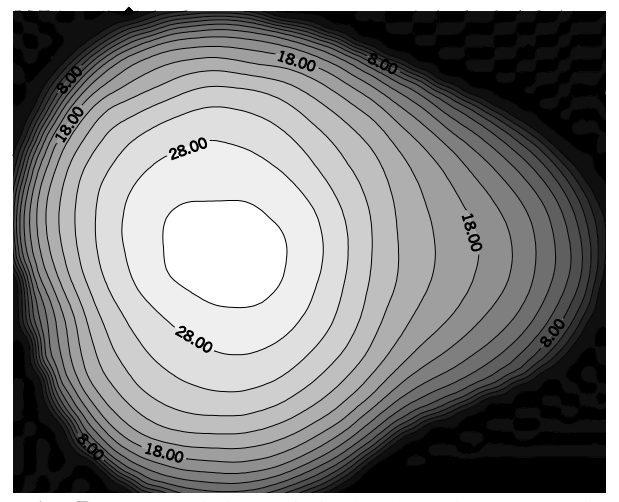

A

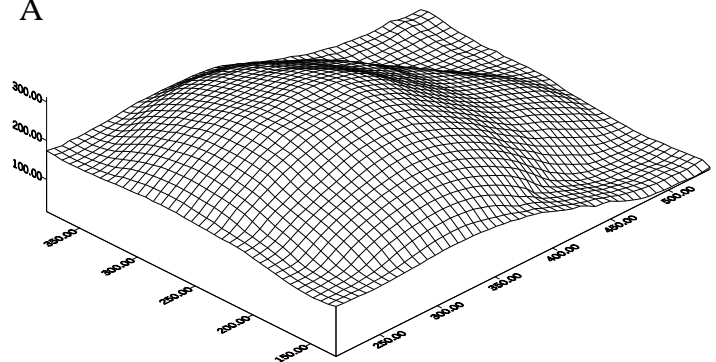

B

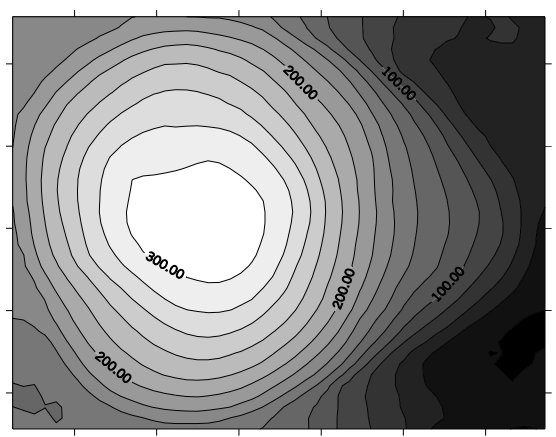

FIGURA 5 - Modelo Digital de Elevação da pêra FIGURA 6 - Modelo Digital de Elevação da pêra gerado pelo Scanner 3D. A. Perspectiva lateral. B. gerado TM de moiré. A. Perspectiva lateral. B. Vista Vista de topo. de topo.

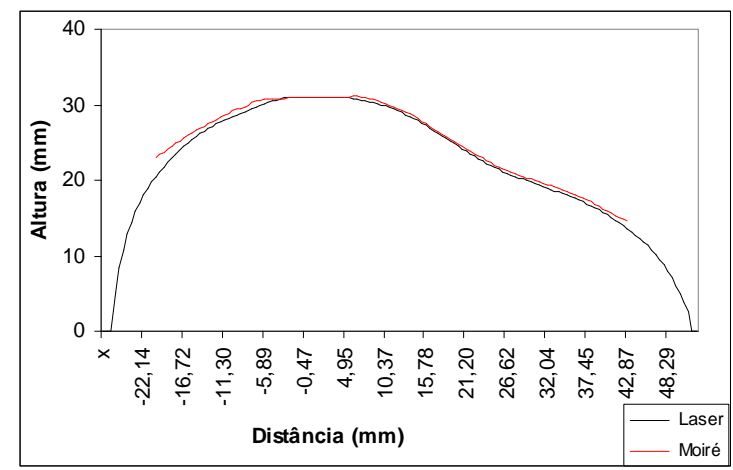

FIGURA 7 - Sobreposição do perfil central dos MDE da pêra produzidos pela TM e Scanner 3D.

TABELA 1 - Comparação entre os MDE da pêra gerados pelo TM de sombra com multiplicação de franjas e pelo Scanner 3D Laser.

\begin{tabular}{ccccc}
\hline & \multicolumn{4}{c}{ Perfil } \\
\cline { 2 - 5 } & E20 & Central & D20 & Média Geral \\
\hline Correlação & 0,9870 & 0,9980 & 0,9583 & 0,9928 \\
\hline $\mathrm{R}^{2}$ & 0,9812 & 0,996 & 0,9317 & 0,9820 \\
\hline $\mathrm{d}$ & 0,9427 & 0,9962 & 0,9195 & 0,9785 \\
\hline
\end{tabular}

Ciênc. agrotec., Lavras, v. 28, n. 1, p. 119-125, jan./fev., 2004 
Os resultados para o perfil D20 foram um pouco menores, comparados ao E20, provavelmente pela existência de ruídos nessa região, que prejudicaram a visualização e digitalização das franjas de moiré, como pode ser verificado na Figura 3.

\section{CONCLUSÕES}

a) A TM de sombra com multiplicação de franjas permitiu a geração do Modelo Digital de Elevação (MDE) da pêra.

b) O MDE reproduz satisfatoriamente a topografia de uma pêra.

c) A TM de sombra com multiplicação de franjas é muito demorada e trabalhosa.

d) A TM de sombra com multiplicação de franjas, dentro das condições do experimento, é indicada por objetos ou regiões relativamente planas.

\section{REFERÊNCIAS BIBLIOGRÁFICAS}

DIRKX, J. J. J.; DECRAEMER, W. F. Automatic calibation method for phase shift shadow moiré interferometry. Applied Optics, Washington, v. 29, n. 10, p. 1474-76, 1990.

GASVIK, K. J. Moiré techniques by means of digital image processing. Applied Optics, Washington, v. 22, n. 23 , p. 3543-3548, set. 1983.

HU, Q. 3-D shape measurement techniques. Disponível em: $\quad$ enhttp://Www.sinc.sunysb.edu/Stu/qhü /Chapter1.htm>. Acesso em: 06 set. 2001 .
MEADOWS, D. M.; JONHSON, W. O.; ALLEN, J. B. Generation of surface contours by moiré patterns. Applied Optics, Washington, v. 9, n. 4, p. 942-947, mar. 1970.

MOORE, D. T.; TRUAX, B. E. Phase-locked moiré fringe analysis for automatic contouring of diffuse surface. Applied Optics, Washington, v. 18, n. 4, p. 9196, mar. 1979.

OSTER, G.; NISHIJIMA, Y. Moiré patterns. Scientific American Resource Library: Readings in the Physics Sciences and Technology, Washington, v. 3, p. 54-63, 1964.

POST, D.; HAN, B.; IFJU, P. High sensitivity moiré: experimental analysis for mechanics and materials. New York: Spring-Verlag, 1994.

TAKASAKI, H. Moiré topography. Applied Optics, Washington, v. 9, n. 6, p. 1452-1457, 1970.

VALERIANO, M. de M. Estimativas de variáveis topográficas por geoprocessamento para modelagem da perda de solo. 1999. 156 f. Tese (Doutorado em Geociências) - Universidade Estadual Paulista, Rio Claro, 1999.

WILLMOT, C. J.; ACKLESON, S. G.; DAVIS, J. J.; FEDDEMA, K. M.; KLINK, D. R. Statistics for the evaluation and comparison of models. Journal Geograph Research, [S.1.], v. 90, p. 8995-9005, 1985. 